\title{
Regularization of a Cauchy problem for the heat equation
}

- Vo Van Au

University of Science, VNU-HCM

Can Tho University of Technology

- Nguyen Hoang Tuan

University of Education, Ho Chi Minh

(Received on $5^{\text {th }}$ December, 2016, accepted on $28^{\text {th }}$ November, 2017)

\begin{abstract}
In this paper, we study a Cauchy problem for the heat equation with linear source in the form

Cauchy data $\varphi^{\varepsilon}$ and $\psi^{\varepsilon}$ satisfying $\left\|\varphi^{\varepsilon}-\varphi\right\|+\left\|\psi^{\varepsilon}-\psi\right\| \leq \varepsilon$ and that $f^{\varepsilon} \quad$ satisfying $\boldsymbol{u}_{t}(x, t)=\boldsymbol{u}_{x x}(x, t)+f(x, t), \boldsymbol{u}(L, t)=\varphi(t), \boldsymbol{u}_{x}(L, t)=\psi(t),(x, t) \in(0, L) \times(0,2 \pi)$. This problem is ill-posed in the sense of Hadamard. To regularize the problem, the truncation method is proposed to solve the problem in the presence of noisy $\left\|f^{\varepsilon}(x, \cdot)-f(x, \cdot)\right\| \leq \varepsilon$. We give some error estimates between the regularized solution and the exact solution under some different a-priori conditions of exact solution.
\end{abstract}

Key words: elliptic equation, ill-posed problem, cauchy problem, regularization method, truncation method

\section{INTRODUCTION}

In this paper, the temperature $\boldsymbol{u}(x, t)$ for $(x, t) \in[0, L] \times[0,2 \pi]$ is sought from known boundary temperature $\boldsymbol{u}(L, t)=\varphi(t)$ and heat flux $\boldsymbol{u}_{x}(L, t)=\psi(t)$ measurements satisfying the following problem:

$\begin{cases}u_{t}(x, t)=u_{x x}(x, t)+f(x, t), & 0<x<L, 0 \leq t \leq 2 \pi, \\ u(L, t)=\varphi(t), & 0 \leq t \leq 2 \pi,\end{cases}$

where $/(t), \varphi, \psi$ are $0 \leq t$ giken functions (usually in $\left.L^{2}(0,2 \pi)\right)$ and $f$ is a given linear heat source which may depend on the independent variables $(x, t)$.

Note that we have no initial condition prescribed at $t=0$ and moreover, the Cauchy data $\varphi$ and $\psi$ are perturbed so as to contain measurement errors in the form of the input noisy Cauchy data $\varphi^{\varepsilon}$ and $\psi^{\varepsilon}$ (also in $\left.L^{2}(0,2 \pi)\right)$ satisfying

$$
\left\|\varphi^{\varepsilon}-\varphi\right\|+\left\|\psi^{\varepsilon}-\psi\right\| \leq \varepsilon,
$$

where $\|\cdot\|$ denotes the $L^{2}(0,2 \pi)$-norm and $\varepsilon>0$ is a small positive number representing the level of noise.
It is well-known that, at least in the linear case, the problem (1) has at most one solution using classical analytical sideways continuation for the parabolic heat equation. The existence of solution also holds, in the case $f=0$. However, the problem is still ill-posed in the sense that the solution, if it exists, does not depend continuously on the data. Any small perturbation in the observation data can cause large errors in the solution $\boldsymbol{u}(x, t)$ for $x \in[0, L)$. Therefore, most classical numerical methods often fail to give an acceptable approximation of the solution. Thus regularization techniques are required to stabilize the solution [3].

In recent years, the homogeneous sideways heat equation, i.e., $f=0$ in the first equation in (1), has been researched by many authors and various methods have been proposed, e.g. the difference regularization method [8], the boundary element Tikhonov regularization method [5], the Fourier method [9], the quasi-reversibility method $[1,6]$, the wavelet, wavelet-

\section{Trang 184}


Galerkin and spectral regularization methods [2, 7], the conjugate gradient method [4], to mention only a few.

To the best of our knowledge, the Cauchy problem for the linear sideways heat equation has not yet been. Therefore, in the present paper, we propose a new method that is based on linear integral equation to regularize problem (1) under two a priori conditions on the exact solution.

As will be shown in next section, for the linear sideways heat problem (1), its solution (exact solution) can be represented as an integral equation which contains some instability terms. In order to restore the stability we replace these instable terms by some regularization ones and show that the solution of our regularized problem converges to the solution of the original linear problem (if such solution exists), as the regularization parameter tends to zero. In the nonhomogeneous problem, we have many choises of stability terms for regularization. However, in the case of non-homogeneous problem, the main solution $u$ is complicated and is defined by a linear integral equation whose the right-hand side depends on the independent variables $(x, t)$. In this paper, we develop a truncation method to solve in a stable manner this linear integral equation.

\section{THE MAIN RESULTS}

Let $\langle\cdot\rangle$ denote the inner product in $L^{2}(0,2 \pi)$, and $\varepsilon>0$ represent the noise level in (2). For $\xi \in L^{2}(0,2 \pi)$, we have the Fourier series $\xi(t)=\sum_{n \in \mathbb{Z}}\langle\xi(t), \exp (-i n t)\rangle \exp (i n t)$, where $\langle\xi(t), \exp (-i n t)\rangle=\frac{1}{2 \pi} \int_{0}^{2 \pi} \xi(t) \exp (-i n t) \mathrm{d} t$. The $L^{2}(0,2 \pi)$-norm of $\xi$ is

$$
\|\xi\|^{2}=2 \pi \sum_{n \in \mathbb{Z}}|\langle\xi(t), \exp (-i n t)\rangle|^{2} .
$$

The principal value of $\sqrt{i n}$ is

$$
\sqrt{i n}= \begin{cases}(1+i) \sqrt{|n| / 2}, & n \geq 0, \\ (1-i) \sqrt{|n| / 2}, & n<0 .\end{cases}
$$

Suppose that the solution of problem (1) is represented as a Fourier series

$$
\boldsymbol{u}(x, t)=\sum_{n \in \mathbb{Z}} \boldsymbol{u}_{n}(x) \exp (\text { int }), \text { with } \boldsymbol{u}_{n}(x)=\langle\boldsymbol{u}(x, t), \exp (-i n t)\rangle=\frac{1}{2 \pi} \int_{0}^{2 \pi} \boldsymbol{u}(x, t) \exp (-i n t) \mathrm{d} t .
$$

From (1), we have the following systems of second-order ordinary differential equations:

$$
\begin{cases}-\frac{\mathrm{d}^{2} \boldsymbol{u}_{n}}{\mathrm{~d} x^{2}}(x)+i n \boldsymbol{u}_{n}(x)=f_{n}(x), & 0<x<L \\ \boldsymbol{u}_{n}(L)=\varphi_{n}=\langle\varphi(t), \exp (-i n t)\rangle, & t \in(0,2 \pi), \\ \frac{\mathrm{d} \boldsymbol{u}_{n}}{\mathrm{~d} x}(L)=\psi_{n}=\langle\psi(t), \exp (-i n t)\rangle, & t \in(0,2 \pi),\end{cases}
$$

where $f_{n}(x)=\langle f(x, t), \exp (-i n t)\rangle=\frac{1}{2 \pi} \int_{0}^{2 \pi} f(x, t) \exp (-i n t) \mathrm{d} t$ for all $n \in \mathbb{Z}$. 
For $n \in \mathbb{Z} \backslash\{0\}$, multiplying the first equation in (5) by $\frac{\sinh ((\tau-x) \sqrt{i n})}{\sqrt{i n}}$ and integrating both sides from $x$ to $L$, we obtain

$\boldsymbol{u}_{n}(x)=\cosh ((L-x) \sqrt{i n}) \boldsymbol{u}_{n}(L)-\frac{\sinh ((L-x) \sqrt{i n})}{\sqrt{\text { in }}} \boldsymbol{u}_{n}^{\prime}(L)-\int_{x}^{L} \frac{\sinh ((\tau-x) \sqrt{\text { in }})}{\sqrt{\text { in }}} f_{n}(\tau) \mathrm{d} \tau, n \in \mathbb{Z} \backslash\{0\}$.

In the case $n=0$, multiplying the first equation in (5) by $\tau-x$ and integrating both sides from $x$ to $L$, we obtain

$$
\boldsymbol{u}_{0}(x)=\boldsymbol{u}_{0}(L)-(L-x) \boldsymbol{u}_{0}^{\prime}(L)-\int_{x}^{L}(\tau-x) f_{0}(\tau) \mathrm{d} \tau .
$$

From (6) - (7) the exact form of $u$ is given by

$$
\begin{aligned}
& \boldsymbol{u}(x, t)=\sum_{n \in \mathbb{Z} \backslash\{0\}}\left[\cosh ((L-x) \sqrt{\text { in }}) \varphi_{n}-\frac{\sinh ((L-x) \sqrt{\text { in }})}{\sqrt{\text { in }}} \psi_{n}-\int_{x}^{L} \frac{\sinh ((\tau-x) \sqrt{\text { in }})}{\sqrt{\text { in }}} f_{n}(\tau) \mathrm{d} \tau\right] \exp (\text { int }) \\
& +\Theta\left(\varphi_{0}, \psi_{0}, f_{0}\right)(x),
\end{aligned}
$$

where $\Theta\left(\varphi_{0}, \psi_{0}, f_{0}\right)(x)=\varphi_{0}-(L-x) \psi_{0}-\int_{x}^{L}(\tau-x) f_{0}(\tau) \mathrm{d} \tau$. In a few sentences, we present a brief introduction Fourier truncated method. From equation (8), it can be observed that $\cosh ((L-x) \sqrt{\text { in }}), \frac{\sinh ((L-x) \sqrt{\text { in }})}{\sqrt{\text { in }}}$ and $\frac{\sinh ((\tau-x) \sqrt{\text { in }})}{\sqrt{\text { in }}}$ are unbounded, as $n$ tends to infinity, so in order to guarantee the convergence of the solution $u$ given by (8), the coefficient $\left(\varphi_{n}, \psi_{n}\right)$ must decay rapidly. But such a decay usually cannot occur for the measured data $\left(\varphi_{n}^{\varepsilon}, \psi_{n}^{\varepsilon}\right)$. Hence, a natural way is to eliminate the high frequencies and consider the solution $u$ for $n \leq N_{\varepsilon}$, where $N_{\varepsilon}$ is a positive integer; this is the so-called Fourier truncated method, and $N_{\varepsilon}$ plays the role of a regularization parameter satisfying $\lim _{\varepsilon \rightarrow 0} N_{\varepsilon}=+\infty$. We define the following two operators:

$$
\begin{aligned}
\boldsymbol{Q}_{N_{\varepsilon}}^{+}(\varphi, \psi, f)(x, t) & =\sum_{|n|>N_{\varepsilon}} \boldsymbol{Q}_{N_{\varepsilon}, n}^{+}(\varphi, \psi, f)(x) \exp (\text { int }) \\
& =\frac{1}{2} \sum_{|n|>N_{\varepsilon}}\left[\exp ((L-x) \sqrt{i n}) \varphi_{n}-\frac{\exp ((L-x) \sqrt{i n})}{\sqrt{i n}} \psi_{n}-\int_{x}^{L} \frac{\exp ((\tau-x) \sqrt{i n})}{\sqrt{i n}} f_{n}(\tau) \mathrm{d} \tau\right] \exp (\text { int }), \\
\boldsymbol{Q}_{N_{\varepsilon}}^{-}(\varphi, \psi, f)(x, t) & =\sum_{|n|>N_{\varepsilon}} \boldsymbol{Q}_{N_{\varepsilon}, n}^{-}(\varphi, \psi, f)(x) \exp (\text { int }) \\
& =\frac{1}{2} \sum_{|n|>N_{\varepsilon}}\left[\exp (-(L-x) \sqrt{\text { in }}) \varphi_{n}+\frac{\exp (-(L-x) \sqrt{i n})}{\sqrt{i n}} \psi_{n}+\int_{x}^{L} \frac{\exp (-(\tau-x) \sqrt{i n})}{\sqrt{i n}} f_{n}(\tau) \mathrm{d} \tau\right] \exp (\text { int }) .
\end{aligned}
$$

To approximate $u$, we introduce the regularized solution

\section{Trang 186}




$$
\begin{aligned}
& \boldsymbol{u}_{N_{\varepsilon}}^{\varepsilon}(x, t)=\sum_{|n| \leq N_{\varepsilon}, n \neq 0}\left[\cosh ((L-x) \sqrt{\text { in }}) \varphi_{n}^{\varepsilon}-\frac{\sinh ((L-x) \sqrt{\text { in }})}{\sqrt{\text { in }}} \psi_{n}^{\varepsilon}-\int_{x}^{L} \frac{\sinh ((\tau-x) \sqrt{\text { in }})}{\sqrt{\text { in }}} f_{n}^{\varepsilon}(\tau) \mathrm{d} \tau\right] \exp (\text { int }) \\
& +\boldsymbol{Q}_{N_{\varepsilon}}^{-}\left(\varphi^{\varepsilon}, \psi^{\varepsilon}, f^{\varepsilon}\right)(x, t)+\Theta\left(\varphi_{0}^{\varepsilon}, \psi_{0}^{\varepsilon}, f_{0}^{\varepsilon}\right)(x) .
\end{aligned}
$$

Our these results would be applied after any necessary minor modifications have been made.

Lemma 1. For $n \in \mathbb{Z} \backslash\{0\}$ and $|n| \leq M$, we have the following inequalities:

$|\cosh (L-x) \sqrt{i n}| \leq \exp \left(\sqrt{\frac{M}{2}}(L-x)\right)$

$|\sinh (\tau-x) \sqrt{i n}| \leq \exp \left(\sqrt{\frac{M}{2}}(\tau-x)\right)$.

Proof. For $n \in \mathbb{Z} \backslash\{0\}, n<M$, one has

$|\cosh (L-x) \sqrt{\text { in }}|=\left|\frac{\exp (L-x) \sqrt{\text { in }}+\exp -(L-x) \sqrt{\text { in }}}{2}\right|$

$$
\begin{aligned}
& \leq \frac{1}{2}|\exp (L-x) \sqrt{\text { in }}|+\frac{1}{2}|\exp -(L-x) \sqrt{\text { in }}| \leq \frac{1}{2} \exp \left(\sqrt{\frac{|n|}{2}}(L-x)\right)+\frac{1}{2} \exp \left(-\sqrt{\frac{|n|}{2}}(L-x)\right) \\
& \leq \frac{1}{2} \exp \left(\sqrt{\frac{M}{2}}(L-x)\right)+\frac{1}{2} \leq \exp \left(\sqrt{\frac{M}{2}}(L-x)\right),
\end{aligned}
$$

and

$$
\begin{aligned}
\left|\frac{\sinh (L-x) \sqrt{\text { in }}}{\sqrt{\text { in }}}\right| & =\left|\frac{\exp (L-x) \sqrt{\text { in }}-\exp (L-x) \sqrt{\text { in }} \mid}{2 \sqrt{\text { in }}}\right| \\
& \leq \frac{\exp (L-x) \sqrt{\text { in }} \mid}{2 \sqrt{|n|}}+\frac{|\exp -(L-x) \sqrt{\text { in }}|}{2 \sqrt{|n|}} \leq \frac{1}{2} \exp \left(\sqrt{\frac{|n|}{2}}(L-x)\right)+\frac{1}{2} \exp \left(-\sqrt{\frac{|n|}{2}}(L-x)\right) \\
& \leq \frac{1}{2} \exp \left(\sqrt{\frac{M}{2}}(L-x)\right)+\frac{1}{2} \leq \exp \left(\sqrt{\frac{M}{2}}(L-x)\right),
\end{aligned}
$$

as required.

Lemma 2. For $|n|>N_{\varepsilon}$, we have

$$
\boldsymbol{Q}_{N_{\varepsilon}, n}^{+}(\varphi, \psi, f)(x)=\frac{1}{2}\left(\boldsymbol{u}_{n}(x)-\frac{\boldsymbol{u}_{n}^{\prime}(x)}{\sqrt{i n}}\right) .
$$

Proof. Differentiating (6) with respect to $x$ gives

$$
-\frac{\boldsymbol{u}_{n}^{\prime}(x)}{\sqrt{\text { in }}}=\sinh ((L-x) \sqrt{\text { in }}) \varphi_{n}-\frac{\cosh ((L-x) \sqrt{i n})}{\sqrt{\text { in }}} \psi_{n}-\int_{x}^{L} \frac{\cosh ((\tau-x) \sqrt{i n})}{\sqrt{\text { in }}} f_{n}(\tau) \mathrm{d} \tau .
$$

Adding (15) to (6), we infer that 


$$
\boldsymbol{u}_{n}(x)-\frac{\boldsymbol{u}_{n}^{\prime}(x)}{\sqrt{\text { in }}}=\exp ((L-x) \sqrt{\text { in }}) \varphi_{n}-\frac{\exp ((L-x) \sqrt{i n})}{\sqrt{\text { in }}} \psi_{n}-\int_{x}^{L} \frac{\exp ((\tau-x) \sqrt{\text { in }})}{\sqrt{\text { in }}} f_{n}(\tau) \mathrm{d} \tau,
$$

from which complete the proof.

The following theorem comes from the regularization $\boldsymbol{u}_{N_{\varepsilon}}$ provides the error estimates in the $L^{2}$-norm when the exact solution belongs to new spaces $\boldsymbol{G}_{\sigma}^{s},(s>0)$. Here $\boldsymbol{G}_{\sigma}^{s}$ is presented by $\boldsymbol{G}_{\sigma}^{s}(0,2 \pi)=\left\{\xi \in L^{2}(0,2 \pi): \sum_{n \in \mathbb{Z}}|n|^{2 s} \exp \sigma \sqrt{2|n|}|\langle\xi(t), \exp (-i n t)\rangle|^{2}<\infty\right\}$,

and this norm is given by

$$
\|\xi\|_{G_{\sigma}^{s}((0,2 \pi)}=\sqrt{|n|^{2 s} \exp \sigma \sqrt{2|n|}\left|\langle\xi(t), \exp (-i n t)\rangle_{L^{2}(0,2 \pi)}\right|^{2}} .
$$

For a Hilbert space $X$, we denote

$$
L^{\infty}(0, L ; X)=\left\{\xi:[0, L] \rightarrow X \mid \underset{0 \leq \tau \leq L}{\operatorname{esssup}}\|\xi(\tau)\|_{X}<\infty\right\},
$$

and

$$
\|\xi\|_{L^{\infty}(0, L ; X)}=\underset{0 \leq \tau \leq L}{\operatorname{ess} \sup }\|\xi(\tau)\|_{X} .
$$

Theorem 1. Assume that problem (1) has a weak solution $\boldsymbol{u} \in C\left([0, T] ; L^{2}(0,2 \pi)\right)$. Choose $N_{\varepsilon}>0$ such that

$$
\lim _{\varepsilon \rightarrow 0} N_{\varepsilon}^{-1}=\lim _{\varepsilon \rightarrow 0}\left[\varepsilon \exp \left(L \sqrt{\frac{N_{\varepsilon}}{2}}\right)\right]=0 .
$$

(a). Suppose that the problem (1) has a solution u satisfying

$$
\|\boldsymbol{u}\|_{L^{\infty}\left(0, L ; \boldsymbol{G}_{L}^{0}(0,2 \pi)\right)}+\left\|\boldsymbol{u}_{x}\right\|_{L^{\infty}\left(0, L ; G_{L}^{0}(0,2 \pi)\right)} \leq E_{1},
$$

for some known constant $E_{1}>0$. Then

$$
\left\|\boldsymbol{u}_{N_{\varepsilon}}^{\varepsilon}(x, \cdot)-\boldsymbol{u}(x, \cdot)\right\| \leq \sqrt{P^{2}+2 \pi E_{1}^{2}} \exp \left(-x \sqrt{\frac{N_{\varepsilon}}{2}}\right),
$$

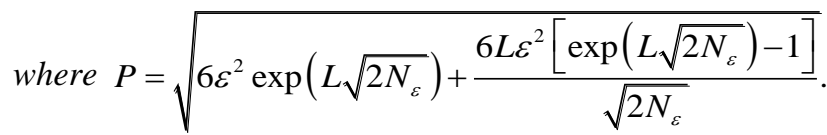

(b). Suppose that the problem (1) has a solution u satisfying

$$
\|\boldsymbol{u}\|_{L^{\infty}\left(0, L ; \boldsymbol{G}_{L}^{r}(0,2 \pi)\right)}+\left\|\boldsymbol{u}_{x}\right\|_{L^{\infty}\left(0, L ; \boldsymbol{G}_{L}^{r}(0,2 \pi)\right)} \leq E_{2}
$$

for $r \geq 0$ and some known constant $E_{2}>0$. Then

$$
\left\|\boldsymbol{u}_{N_{\varepsilon}}^{\varepsilon}(x, \cdot)-\boldsymbol{u}(x, \cdot)\right\| \leq \sqrt{P^{2}+2 \pi N_{\varepsilon}^{-2 r} E_{2}^{2}} \exp \left(-x \sqrt{\frac{N_{\varepsilon}}{2}}\right) .
$$

Corollary 1. Let us choose $N_{\varepsilon}=\frac{2}{(L+\delta)^{2}} \ln ^{2}\left(\frac{1}{\varepsilon}\right)$ for $\delta>0$ then

\section{Trang 188}


Estimate in (22) is calculated as follows

$$
\left\|\boldsymbol{u}_{N_{\varepsilon}}^{\varepsilon}(x, \cdot)-\boldsymbol{u}(x, \cdot)\right\| \leq \sqrt{R^{2}+2 \pi E_{1}^{2}} \varepsilon^{\frac{x}{L+\delta}},
$$

where $R=\sqrt{6 \varepsilon^{\frac{2 \delta}{L+\delta}}+6 L\left(\varepsilon^{\frac{2 \delta}{L+\delta}}-\varepsilon^{2}\right)\left[\frac{2}{L+\delta} \ln \left(\frac{1}{\varepsilon}\right)\right]^{-1}}$.

2. Estimate in (24) is calculated as follows

$$
\left\|\boldsymbol{u}_{N_{\varepsilon}}^{\varepsilon}(x, \cdot)-\boldsymbol{u}(x, \cdot)\right\| \leq \sqrt{R^{2}+2 \pi\left[\frac{\sqrt{2}}{L+\delta} \ln \left(\frac{1}{\varepsilon}\right)\right]^{-4 r} E_{2}^{2}} \varepsilon^{\frac{x}{L+\delta}} .
$$

Proof of the Theorem 1. The proof is divided into two parts.

Part a. Estimate the error (22) between the regularization $\boldsymbol{u}_{N_{\varepsilon}}^{\varepsilon}$ and the exact solution $\boldsymbol{u}$ with a priori (21).

We rewrite $\boldsymbol{u}$ as

$$
\begin{aligned}
\boldsymbol{u}(x, t)= & \sum_{|n| \leq N_{\varepsilon}, n \neq 0}\left[\cosh ((L-x) \sqrt{\text { in }}) \varphi_{n}-\frac{\sinh ((L-x) \sqrt{i n})}{\sqrt{i n}} \psi_{n}-\int_{x}^{L} \frac{\sinh ((\tau-x) \sqrt{i n})}{\sqrt{i n}} f_{n}(\tau) \mathrm{d} \tau\right] \exp (i n t) \\
& +\Theta\left(\varphi_{0}, \psi_{0}, f_{0}\right)(x)+\boldsymbol{Q}_{N_{\varepsilon}}^{+}(\varphi, \psi, f)(x, t)+\boldsymbol{Q}_{N_{\varepsilon}}^{-}(\varphi, \psi, f)(x, t) .
\end{aligned}
$$

From (11) and (27), thanks to Parseval's relation, we obtain

$$
\begin{aligned}
\left\|\boldsymbol{u}_{N_{\varepsilon}}^{\varepsilon}(x, \cdot)-\boldsymbol{u}(x, \cdot)\right\|^{2}= & \underbrace{2 \pi \sum_{|n| \leq N_{\varepsilon}, n \neq 0}\left|\boldsymbol{u}_{N_{\varepsilon}, n}^{\varepsilon}(x)-\boldsymbol{u}_{n}(x)\right|^{2}}_{:=J_{1}(x)}+\underbrace{4 \pi \sum_{|n|>N_{\varepsilon}}\left|\boldsymbol{Q}_{N_{\varepsilon}, n}^{-}\left(\varphi^{\varepsilon}, \psi^{\varepsilon}, f^{\varepsilon}\right)(x)-\boldsymbol{Q}_{N_{\varepsilon}, n}^{-}(\varphi, \psi, f)(x)\right|^{2}}_{:=J_{2}(x)} \\
& +\underbrace{2 \pi\left|\Theta\left(\varphi_{n}^{\varepsilon}, \psi_{n}^{\varepsilon}, f_{n}^{\varepsilon}\right)(x)-\Theta\left(\varphi_{0}, \psi_{0}, f_{0}\right)(x)\right|^{2}}_{:=J_{3}(x)}+\underbrace{4 \pi \sum_{|n|>N_{\varepsilon}}\left|\boldsymbol{Q}_{N_{\varepsilon}, n}^{+}(\varphi, \psi, f)(x)\right|^{2}}_{:=J_{4}(x)} .
\end{aligned}
$$

We now apply Lemma 1 and using the Holder's inequality, we have

$$
\begin{aligned}
J_{1}(x) \leq & 6 \pi \sum_{|n| \leq N_{\varepsilon}, n \neq 0}\left[|\cosh ((L-x) \sqrt{i n})|^{2}\left|\varphi_{n}^{\varepsilon}-\varphi_{n}\right|^{2}+\left|\frac{\sinh ((L-x) \sqrt{i n})}{\sqrt{i n}}\right|^{2}\left|\psi_{n}^{\varepsilon}-\psi_{n}\right|^{2}\right] \\
& +6 \pi \sum_{|n| \leq N_{\varepsilon}, n \neq 0}\left|\int_{x}^{L} \frac{\sinh ((\tau-x) \sqrt{i n})}{\sqrt{i n}}\left(f_{n}^{\varepsilon}(\tau)-f_{n}(\tau)\right) \mathrm{d} \tau\right|^{2} \\
\leq & 6 \pi \sum_{|n| \leq N_{\varepsilon}, n \neq 0}\left[\exp \left((L-x) \sqrt{2 N_{\varepsilon}}\right)\left|\varphi_{n}^{\varepsilon}-\varphi_{n}\right|^{2}+\exp \left((L-x) \sqrt{2 N_{\varepsilon}}\right)\left|\psi_{n}^{\varepsilon}-\psi_{n}\right|^{2}\right] \\
& +6 \pi \sum_{|n| \leq N_{\varepsilon}, n \neq 0}\left[(L-x) \int_{x}^{L} \exp \left((\tau-x) \sqrt{2 N_{\varepsilon}}\right)\left|f_{n}^{\varepsilon}(\tau)-f_{n}(\tau)\right|^{2} \mathrm{~d} \tau\right],
\end{aligned}
$$

where we have used the elementary inequality $(a+b+c)^{2} \leq 3\left(a^{2}+b^{2}+c^{2}\right)$.

Similarly, the second equation $J_{2}(x)$ writes 


$$
\begin{aligned}
J_{2}(x) \leq 12 \pi \sum_{|n|>N_{\varepsilon}} & {\left[|\exp (-(L-x) \sqrt{i n})|^{2}\left|\varphi_{n}^{\varepsilon}-\varphi_{n}\right|^{2}+\left|\frac{\exp (-(L-x) \sqrt{i n})}{\sqrt{i n}}\right|^{2}\left|\psi_{n}^{\varepsilon}-\psi_{n}\right|^{2}\right] } \\
& +12 \pi \sum_{|n|>N_{\varepsilon}}\left|\int_{x}^{L} \frac{\exp (-(\tau-x) \sqrt{i n})}{\sqrt{i n}}\left(f_{n}^{\varepsilon}(\tau)-f_{n}(\tau)\right) \mathrm{d} \tau\right|^{2} \\
\leq & 12 \pi \sum_{|n|>N_{\varepsilon}}\left[\exp \left((L-x) \sqrt{2 N_{\varepsilon}}\right)\left|\varphi_{n}^{\varepsilon}-\varphi_{n}\right|^{2}+\exp \left((L-x) \sqrt{2 N_{\varepsilon}}\right)\left|\psi_{n}^{\varepsilon}-\psi_{n}\right|^{2}\right] \\
& +12 \pi \sum_{|n|>N_{\varepsilon}}\left[(L-x) \int_{x}^{L} \exp \left((\tau-x) \sqrt{2 N_{\varepsilon}}\right)\left|f_{n}^{\varepsilon}(\tau)-f_{n}(\tau)\right|^{2} \mathrm{~d} \tau\right] .
\end{aligned}
$$

Thanks to Holder's inequality and using the basic inequality $e^{a} \geq a, \forall a>0$, we deduce that

$$
\begin{aligned}
J_{3}(x)= & 6 \pi\left[\left|\varphi_{0}^{\varepsilon}-\varphi_{0}\right|^{2}+(L-x)^{2}\left|\psi_{0}^{\varepsilon}-\psi_{0}\right|^{2}+(L-x) \int_{x}^{L}(\tau-x)^{2}\left|f_{0}^{\varepsilon}(\tau)-f_{0}(\tau)\right|^{2} \mathrm{~d} \tau .\right] \\
\leq & 6 \pi\left[\exp \left((L-x) \sqrt{2 N_{\varepsilon}}\right)\left|\varphi_{0}^{\varepsilon}-\varphi_{0}\right|^{2}+\exp \left((L-x) \sqrt{2 N_{\varepsilon}}\right)\left|\psi_{0}^{\varepsilon}-\psi_{0}\right|^{2}\right] \\
& +6 \pi\left[(L-x) \int_{x}^{L} \exp \left((\tau-x) \sqrt{2 N_{\varepsilon}}\right)\left|f_{0}^{\varepsilon}(\tau)-f_{0}(\tau)\right|^{2} \mathrm{~d} \tau\right] .
\end{aligned}
$$

Using Lemma 2, easy calculations show that

$$
\begin{aligned}
J_{4}(x)=4 \pi \sum_{|n|>N_{\varepsilon}} & \left|\boldsymbol{Q}_{N_{\varepsilon}, n}^{+}(\varphi, \psi, f)(x)\right|^{2}=4 \pi \sum_{|n|>N_{\varepsilon}}\left|\frac{1}{2}\left(\boldsymbol{u}_{n}(x)-\frac{\boldsymbol{u}_{n}^{\prime}(x)}{\sqrt{i n}}\right)\right|^{2} \\
& \leq \pi \sum_{|n|>N_{\varepsilon}}\left|\exp (-x \sqrt{i n})\left(\exp (x \sqrt{i n}) \boldsymbol{u}_{n}(x)-\exp (x \sqrt{i n}) \frac{\boldsymbol{u}_{n}^{\prime}(x)}{\sqrt{i n}}\right)\right|^{2} \\
& \leq \pi \sum_{|n|>N_{\varepsilon}}|\exp (-x \sqrt{i n})|^{2}\left|\exp (x \sqrt{i n}) \boldsymbol{u}_{n}(x)-\exp (x \sqrt{i n}) \frac{\boldsymbol{u}_{n}^{\prime}(x)}{\sqrt{i n}}\right|^{2} \\
& \leq 2 \pi \exp \left(-x \sqrt{2 N_{\varepsilon}}\right)\left[\sum_{|n|>N_{\varepsilon}} \exp (L \sqrt{2|n|})\left|\boldsymbol{u}_{n}(x)\right|^{2}+\sum_{|n|>N_{\varepsilon}} \exp (L \sqrt{2|n|})\left|\frac{\boldsymbol{u}_{n}^{\prime}(x)}{\sqrt{i n}}\right|^{2}\right] \\
& \leq 2 \pi \exp \left(-x \sqrt{2 N_{\varepsilon}}\right)\left[\|\boldsymbol{u}\|_{L^{\infty}}^{2}\left(0, L ; \mathbf{G}_{L}^{0}(0,2 \pi)\right)+\left\|\boldsymbol{u}_{x}\right\|_{L^{\infty}\left(0, L ; \mathbf{G}_{L}^{0}(0,2 \pi)\right)}^{2}\right] .
\end{aligned}
$$

Combining (28), (29), (30), (31) and (32) we infer

$$
\begin{aligned}
\left\|\boldsymbol{u}_{N_{\varepsilon}}^{\varepsilon}(x, \cdot)-\boldsymbol{u}(x, \cdot)\right\|^{2} \leq & 6 \exp \left((L-x) \sqrt{2 N_{\varepsilon}}\right)\left[\left\|\varphi^{\varepsilon}-\varphi\right\|^{2}+\left\|\psi^{\varepsilon}-\psi\right\|^{2}+(L-x) \int_{x}^{L} \exp \left((\tau-L) \sqrt{2 N_{\varepsilon}}\right)\left\|f^{\varepsilon}(\tau, \cdot)-f(\tau, \cdot)\right\|^{2} \mathrm{~d} \tau\right] \\
& +2 \pi \exp \left(-x \sqrt{2 N_{\varepsilon}}\right)\left[\|\boldsymbol{u}\|_{L^{\circ}}^{2}\left(0, L \mathbf{G}_{L}^{0}(0,2 \pi)\right)+\left\|\boldsymbol{u}_{x}\right\|_{\left.L^{\circ}\left(0, L ; \mathbf{G}_{L_{(0,2}^{0}}^{2}\right)\right)}^{L}\right] \\
\leq & 6 \exp \left((L-x) \sqrt{2 N_{\varepsilon}}\right)\left[\varepsilon^{2}+L \varepsilon^{2} \int_{x}^{L} \exp \left((\tau-L) \sqrt{2 N_{\varepsilon}}\right) \mathrm{d} \tau\right]+2 \pi \exp \left(-x \sqrt{2 N_{\varepsilon}}\right) E_{1}^{2} \\
\leq & 6 \exp \left((L-x) \sqrt{2 N_{\varepsilon}}\right)\left[\varepsilon^{2}+\frac{L \varepsilon^{2}}{\sqrt{2 N_{\varepsilon}}}\left(1-\exp \left((x-L) \sqrt{2 N_{\varepsilon}}\right)\right)\right]+2 \pi \exp \left(-x \sqrt{2 N_{\varepsilon}}\right) E_{1}^{2},(33)
\end{aligned}
$$

which can be rewritten as

\section{Trang 190}




$$
\left\|\boldsymbol{u}_{N_{\varepsilon}}^{\varepsilon}(x, \cdot)-\boldsymbol{u}(x, \cdot)\right\| \leq\left[6 \varepsilon^{2} \exp \left(L \sqrt{2 N_{\varepsilon}}\right)+\frac{6 L \varepsilon^{2}\left[\exp \left(L \sqrt{2 N_{\varepsilon}}\right)-1\right]}{\sqrt{2 N_{\varepsilon}}}+2 \pi E_{1}^{2}\right]^{1 / 2} \exp \left(-x \sqrt{\frac{N_{\varepsilon}}{2}}\right)
$$

(34)

Part (b). Estimate the error (24) between the regularization $\boldsymbol{u}_{N_{\varepsilon}}^{\varepsilon}$ and the exact solution $\boldsymbol{u}$ with a priori (23).

By an argument analogous to the previous one, the estimates of $J_{1}(x), J_{2}(x), J_{3}(x)$ in the proof of part (a) remains valid. Also, replace $J_{4}(x)$ by following estimate

$$
\begin{aligned}
J_{4}(x) & =4 \pi \sum_{|n|>N_{\varepsilon}}\left|\boldsymbol{Q}_{N_{\varepsilon}, n}^{+}(\varphi, \psi, f)(x)\right|^{2}=4 \pi \sum_{|n|>N_{\varepsilon}}\left|\frac{1}{2}\left(\boldsymbol{u}_{n}(x)-\frac{\boldsymbol{u}_{n}^{\prime}(x)}{\sqrt{i n}}\right)\right|^{2} \\
& \leq\left.\left.\pi \sum_{|n|>N_{\varepsilon}}|| n\right|^{-r} \exp (-x \sqrt{i n})\left(|n|^{r} \exp (x \sqrt{i n}) \boldsymbol{u}_{n}(x)-|n|^{r} \exp (x \sqrt{i n}) \frac{\boldsymbol{u}_{n}^{\prime}(x)}{\sqrt{i n}}\right)\right|^{2} \\
& \leq\left.\pi \sum_{|n|>N_{\varepsilon}}|n|^{-2 r}|\exp (-x \sqrt{i n})|^{2}|| n\right|^{r} \exp (x \sqrt{i n}) \boldsymbol{u}_{n}(x)-\left.|n|^{r} \exp (x \sqrt{i n}) \frac{\boldsymbol{u}_{n}^{\prime}(x)}{\sqrt{i n}}\right|^{2} \\
& \leq 2 \pi N_{\varepsilon}^{-2 r} \exp \left(-x \sqrt{2 N_{\varepsilon}}\right)\left[\sum_{|n|>N_{\varepsilon}}|n|^{2 r} \exp (L \sqrt{2|n|})\left|\boldsymbol{u}_{n}(x)\right|^{2}+\sum_{n>N_{\varepsilon}}|n|^{2 r} \exp (L \sqrt{2|n|})\left|\frac{\boldsymbol{u}_{n}^{\prime}(x)^{2}}{\sqrt{i n}}\right|\right] \\
& \leq 2 \pi N_{\varepsilon}^{-2 r} \exp \left(-x \sqrt{2 N_{\varepsilon}}\right)\left[\|\boldsymbol{u}\|_{L^{\infty}}^{2}\left(0, L ; G_{L}^{r}(0,2 \pi)\right)+\left\|\boldsymbol{u}_{x}\right\|_{L^{\infty}\left(0, L ; \boldsymbol{G}_{L}^{r}(0,2 \pi)\right)}^{2}\right] .
\end{aligned}
$$

Combining (28), (29), (30), (31) and (35), we get

We obtain

$$
\begin{aligned}
\left\|\boldsymbol{u}_{N_{\varepsilon}}^{\varepsilon}(x, \cdot)-\boldsymbol{u}(x, \cdot)\right\|^{2} \leq & 6 \exp \left((L-x) \sqrt{2 N_{\varepsilon}}\right)\left[\left\|\varphi^{\varepsilon}-\varphi\right\|^{2}+\left\|\psi^{\varepsilon}-\psi\right\|^{2}+(L-x) \int_{x}^{L} \exp \left((\tau-L) \sqrt{2 N_{\varepsilon}}\right)\left\|f^{\varepsilon}(\tau, \cdot)-f(\tau, \cdot)\right\|^{2} \mathrm{~d} \tau\right] \\
& +2 \pi N_{\varepsilon}^{-2 r} \exp \left(-x \sqrt{2 N_{\varepsilon}}\right)\left[\|\boldsymbol{u}\|_{L^{\infty}}^{2}\left(0, L ; \boldsymbol{G}_{L}^{r}(0,2 \pi)\right)+\left\|\boldsymbol{u}_{x}\right\|_{L^{\circ}\left(0, L ; \boldsymbol{G}_{L}^{r}(0,2 \pi)\right)}^{2}\right] \\
\leq & 6 \exp \left((L-x) \sqrt{2 N_{\varepsilon}}\right)\left[\varepsilon^{2}+L \varepsilon^{2} \int_{x}^{L} \exp \left((\tau-L) \sqrt{2 N_{\varepsilon}}\right) \mathrm{d} \tau\right]+2 \pi N_{\varepsilon}^{-2 r} \exp \left(-x \sqrt{2 N_{\varepsilon}}\right) E_{2}^{2} \\
\leq & 6 \exp \left((L-x) \sqrt{2 N_{\varepsilon}}\right)\left[\varepsilon^{2}+\frac{L \varepsilon^{2}}{\sqrt{2 N_{\varepsilon}}}\left(1-\exp \left((x-L) \sqrt{2 N_{\varepsilon}}\right)\right)\right]+2 \pi N_{\varepsilon}^{-2 r} \exp \left(-x \sqrt{2 N_{\varepsilon}}\right) E_{2}^{2} . \\
\left\|\boldsymbol{u}_{N_{\varepsilon}}^{\varepsilon}(x, \cdot)-\boldsymbol{u}(x, \cdot)\right\| \leq & {\left[6 \varepsilon^{2} \exp \left(L \sqrt{2 N_{\varepsilon}}\right)+\frac{6 L \varepsilon^{2}\left[\exp \left(L \sqrt{2 N_{\varepsilon}}\right)-\exp \left(x \sqrt{2 N_{\varepsilon}}\right)\right]}{\sqrt{2 N_{\varepsilon}}}+2 \pi N_{\varepsilon}^{-2 r} E_{2}^{2}\right]^{1 / 2} \exp \left(-x \sqrt{\frac{N_{\varepsilon}}{2}}\right) }
\end{aligned}
$$

This completes the proof of the theorem.

\section{CONCLUSION}

In this paper, the Cauchy problem for the heat equation has been solved by employing the truncation

method for a resulting linear integral equation. Convergence and stability estimates, as the regularization parameter tends to zero, are proved. 


\section{Chỉnh hóa bài toán Cauchy cho phương trình nhiệt}

- Võ Văn Âu

Trường Đại học Khoa học Tự nhiên, ĐHQG-HCM

Trường Đại học Kỹ thuật Công nghệ Cần Thơ

- Nguyễn Hoàng Tuấn

Trường Đại học Sư phạm Thành phố Hồ Chí Minh

\section{TÓM TẮT}

Trong bài báo này, chúng tôi nghiên cứu bài toán Cauchy cho phuoong trình nhiệt với hàm nguồn tuyến tinh thóa phuong trinh: $\boldsymbol{u}_{t}(x, t)=\boldsymbol{u}_{x x}(x, t)+f(x, t), \boldsymbol{u}(L, t)=\varphi(t), \boldsymbol{u}_{x}(L, t)=\psi(t),(x, t) \in(0, L) \times(0,2 \pi)$. Đây là bài toán không chỉnh theo nghĩa của Hadamard. Để chỉnh hóa bài toán này, phuoong pháp chặt cụt được đề xuất để giải quyết bài toán trong

truờng hợp dũ liệu Cauchy $\varphi, \psi$ và hàm nguồn $f$ bị nhiễu bởi $\varphi^{\varepsilon}, \psi^{\varepsilon} \quad v \grave{a} \quad f^{\varepsilon}$ thỏa mãn $\left\|\varphi^{\varepsilon}-\varphi\right\|+\left\|\psi^{\varepsilon}-\psi\right\| \leq \varepsilon \quad$ và $\quad\left\|f^{\varepsilon}(x, \cdot)-f(x, \cdot)\right\| \leq \varepsilon$. Chúng tôi đưa ra các đánh giá sai số giũa nghiệm chỉnh hóa và nghiệm chính xác duoói một số tính trơn khác nhau của nghiệm chính xác.

Tù khóa: phuoong trình Eliptic, bài toán không chỉnh, bài toán Cauchy, phuoong pháp chỉnh hóa, phuoong pháp chặt cụt

\section{TÀI LIẸU THAM KHẢO}

[1]. L. Elden, Approximations for a Cauchy problem for the heat equation, Inverse Problems, 3, 263-273 (1987)

[2]. L. Elden, F. Berntsson, T. Reginska, Wavelet and Fourier methods for solving the sideways heat equation, SIAM J. Sci. Comput., 21, 2187-2205 (2000).

[3]. D.N. Hao, Methods for Inverse Heat Conduction Problems, Peter Lang, Frankfurt am Main (1998).

[4]. D.N. Hao, P.X. Thanh, D. Lesnic, B.T. Johansson, A boundary element method for a multidimensional inverse heat conduction problem, Int. J. Computer Math. 89, 1540-1554 (2012).

[5]. D. Lesnic, L. Elliott, D.B. Ingham, Application of the boundary element method to inverse heat conduction problems, Int. J. Heat Mass Transfer, 39, 1503-1517 (1996).
[6]. J.C. Liu, T. Wei, A quasi-reversibility regularization method for an inverse heat conduction problem without initial data, Appl. Math. Comput., 219, 10866-10881 (2013).

[7]. T. Reginska, L. Elden, Solving the sideways heat equation by a wavelet-Galerkin method, Inverse Problems, 13, 1093-1106 (1997).

[8]. X.T. Xiong, C.L. Fu, H.F. Li, Central difference method of a non-standard inverse heat conduction problem for determining surface heat flux from interior observations, Appl. Math. Comput. 173, 1265-1287 (2006).

[9]. X.T. Xiong, C.L. Fu, H.F. Li, Fourier regularization method of a sideways heat equation for determining surface heat flux, J. Math. Anal. Appl. 317, 331348 (2006).

\section{Trang 192}

